\title{
ON THE FRAMIZATION OF KNOT ALGEBRAS
}

\author{
JESÚS JUYUMAYA AND SOFIA LAMBROPOULOU
}

\begin{abstract}
This paper presents results on the framization of some knot algebras, defined by the authors. We explain the motivations of the concept of framization, coming from the YokonumaHecke algebras, as well as recent results on the framization of the Temperley-Lieb algebra. Finally, we propose framizations for other knot algebras such as the BMW algebra, the $B$-type related Hecke algebras and the singular Hecke algebra.
\end{abstract}

\section{INTRODUCTION}

Modular framization (or simply framization) is a mechanism proposed recently by the authors and it consists in constructing a non-trivial extension of a knot algebra via the addition of framing generators. In this way we obtain a new algebra which is related to framed braids and framed knots.

By knot algebra we mean an algebra that is applied to the construction of invariants of knots and links. We are focused on the framization of those knot algebras that define invariants of knots via the Jones' construction. More precisely, such a knot algebra $A$ is a triplet $(A, \pi, \tau)$, where $\pi$ is a representation of a braid group in $A$ and $\tau$ a Markov trace defined on $A$. The invariant obtained by the knot algebra $A$ is constructed essentially from the composition $\tau \circ \pi$ after re-scaling and normalizing $\tau$ according to the braid equivalence in the given braid category. In Table 1 we list some knot algebras with related knot invariants.

\begin{aligned} Knot algebra & Invariant \\ \hline Temperley-Lieb algebra & Jones polynomial \& bracket polynomial \\ Iwahori-Hecke algebra & HOMFLYPT polynomial \\ BMW algebra & Kauffman polynomial \\ B-type \& affine Hecke algebras & Lambropoulou invariants \\ Singular Hecke algebra & Kauffman-Vogel \& Paris-Rabenda invariants \\ Rook algebra & Alexander polynomial \end{aligned}

TABLE 1. Examples of knot algebras.

The inspiring example of framization is the so-called Yokonuma-Hecke algebra. Indeed, this algebra corresponds to a framization of the Iwahori-Hecke algebra. The Yokonuma-Hecke algebra appears in the field of group theory and was defined by Yokonuma $\mathrm{YO}$ as a generalization of the Iwahori-Hecke algebra. More precisely, he considered the centralizer algebra of the permutation

2010 Mathematics Subject Classification. 57M27, 57M25, 20F38, 20F36, $20 \mathrm{C} 08$.

Key words and phrases. framed links, framed braids, Yokonuma-Hecke algebra, Markov trace, knot invariants, singular links, Temperley-Lieb algebra, BMW algebra, B-type Hecke algebras, affine Hecke algebra, singular Hecke algebra.

This research has been co-financed by the European Union (European Social Fund - ESF) and Greek national funds through the Operational Program "Education and Lifelong Learning" of the National Strategic Reference Framework (NSRF) - Research Funding Program: THALIS. Moreover, the first author was partially supported by Fondecyt 1141254 and Dipuv No. 1/2011. 
representation associated to any finite Chevalley group with respect to one maximal unipotent subgroup. Then, in analogy to the classical presentation of the Iwahori-Hecke algebra, he found a presentation of the Yokonuma-Hecke algebra by generators and relations [Yo, Theorem 1].

In [Ju1, JK] another presentation of the Yokonuma-Hecke algebra was established (Definition 1). By using this new presentation, the Yokonuma-Hecke algebra $\mathrm{Y}_{d, n}(u)$ was defined for any positive integers $n$ and $d$ and a fixed non-zero complex number $u$. Then, $\mathrm{Y}_{d, n}(u)$ could be naturally viewed as a quotient of the framed braid group $\mathcal{F}_{n}$ or as an algebra obtained from the Iwahori-Hecke algebra $\mathrm{H}_{n}(u)$ by adding framing generators and by replacing the Hecke algebra quadratic relation by a quadratic relation which also involves intrinsically the framing generators (Eq. 2.3). For $d=1$ the algebra $\mathrm{Y}_{d, n}(u)$ coincides with the algebra $\mathrm{H}_{n}(u)$.

Further, a Markov trace tr was constructed by the first author on the algebras $\mathrm{Y}_{d, n}(u)$, the 'Juyumaya trace', [Ju2] with parameters $z, x_{1}, \ldots, x_{d-1}$, using an appropriate inductive linear basis. Parameter $z$ takes care of the braiding, while parameters $x_{1}, \ldots, x_{d-1}$ take care of the framing. For $d=1$ the trace tr coincides with the well-known Ocneanu trace $\tau$ on the algebras $\mathrm{H}_{n}(u)$, from which the 2-variable Jones or HOMFLYPT polynomial (denoted here Homflypt) for oriented links was extracted $\mathrm{JO}$.

Then, in order to obtain link invariants via the trace tr, the ' $\mathrm{E}-$ condition' needed to be imposed on the framing parameters $x_{1}, \ldots, x_{d-1}$ (see Eq. 3.2) for re-scaling tr in order that it conforms with negative stabilization (cf. [JL5). The trace tr is the only known Markov trace on knot algebras that does not re-scale directly. As it was shown by Gérardin (cf. Appendix [JL5]) solutions of the 'E-system' (3.3) are parametrized by the non-empty subsets of $\mathbb{Z} / d \mathbb{Z}$.

Subsequently, for any solution of the E-system, tr yielded an invariant for framed knots [JL5], for classical knots [JL4] and for singular knots [JL3]. Moreover, the algebras $\mathrm{Y}_{d, n}(u)$ with the trace tr seem to relate naturally to the domain of transverse knots [CJJKL. All these invariants are still under investigation CL, CJJKL, especially as to how they compare with the Homflypt polynomial. In [CL] it is shown that only in trivial cases the classical link invariants coincide with the Homflypt polynomial. By construction these invariants are at least as strong as the Homflypt polynomial, however computational evidence indicates that they are topologically equivalent. This is not easy to see either by algebraic or by diagrammatic methods. In CJJKL some conjectures are stated in this direction. Still, we believe that it is remarkable that one can obtain invariants for all these different knot categories from a single algebra.

The Yokonuma-Hecke algebras, equipped with a Markov trace, are interesting on their own right. Their representation theory has been studied thoroughly in [Th, C-PA1]. In particular, in [C-PA1] a completely combinatorial approach is taken to the subject.

All these results are presented in Sections 2, 3 and 4. The above comprise our motivation for constructing framizations of other knot algebras. In this paper we present possible framizations of most of the algebras listed in Table 1. Up to Section 4.5 the paper is mostly a survey of results but from there on it continues as an announcement of new results, some by the authors, some by the authors with co-authors and some by other authors.

In Section 5 we present three quotients of the Yokonuma-Hecke algebra $\mathrm{Y}_{d, n}(u)$ as possible framizations of the Temperley-Lieb algebra. For each one we present necessary and sufficient conditions for the trace tr to pass through to the quotient algebra and we discuss related unoriented knot invariants GJKL1, GJKL2]. For the first quotient, the Yokonuma-Temperley-Lieb $\mathrm{YTL}_{d, n}(u)$, the ideal is similar to the one in the classical case. Then, as it turns out, the trace $\operatorname{tr}$ passes to $\mathrm{YTL}_{d, n}(u)$ only if the trace parameters $x_{i}$ are $d^{\text {th }}$ roots of unity. In this case we recover the Jones polynomial. See GJKL1. The second candidate, $\mathrm{FTL}_{d, n}(u)$, which we select as the 'Framization of the Temperley-Lieb algebra', has the property that the conditions on the $x_{i}$ 's so that tr passes through to the quotient, include explicitely all solutions of the E-system mentioned 
above. Finally, the conditions on the trace parameters for the third candidate, the Complex Reflection Temperley-Lieb algebra $\mathrm{CTL}_{d, n}(u)$, involve only parameter $z$ and not the framing parameters $x_{1}, \ldots, x_{d-1}$. So, in order to obtain knot invariants from the algebra $\mathrm{CTL}_{d, n}(u)$ we have to impose the E-condition (Eq. 3.2). It follows that the knot invariants we obtain coincide with those from $\mathrm{FTL}_{d, n}(u)$. The main disadvantage of $\mathrm{CTL}_{d, n}(u)$ is that is is unnecessarily large for our purposes.

In Section 6 we propose framizations for the Hecke algebra of $B$-type, for the cyclotomic Hecke algebras of $B$-type, and for the generalized Hecke algebra of $B$-type, which is isomorphic to the affine Hecke algebra of $A$-type. These definitions were first given in [JL6]. All these algebras are related to the knot theory of the solid torus [La1, GL, La2]. These $B$-type framizations are further studied in [C-PA2], where Markov traces are also constructed and a corresponding E-condition is given.

In Section 7 we propose a framization of the Birman-Murakami-Wenzl or simply BMW algebra [BW,, $\mathrm{Mu}$, which is related to the Kauffman polynomial invariant of knots [Ka1]. This framization was introduced and further studied in [JL6].

Finally, in Section 8 we propose a framization of the singular Hecke algebra [PR], which is related to the invariants of Kauffman-Vogel [KV] and Paris-Rabenda [PR]. This was introduced in [JL6].

All these framization knot algebras are related to the framed braid group and they are of interest to algebraists. There are many more other knot algebras, such as other quotients of the classical braid group, quotients of the virtual braid group [Ka2, KL], or the Rook algebra [BRY] which is

related to the Alexander polynomial. For all these one could construct appropriate framization counterparts.

Acknowledgments We would like to thank the Referee for the very careful reading and for the very interesting comments.

\section{Notations AND BACKGROUnd}

1.1. Along the paper the term algebra means a $\mathbb{C}$-associative algebra with unity denoted 1 , where as usual $\mathbb{C}$ denotes the field of complex numbers. Notice that $\mathbb{C}$ can be regarded as included in the algebra as a central subalgebra. We also denote by $\mathbb{C} G$ the group algebra of a group $G$.

1.2. The letters $n$ and $d$ denote two positive integers. We denote by $S_{n}$ the symmetric group on $n$-symbols and by $s_{i}$ the elementary transposition $(i, i+1)$. Let $B_{n}$ be the classical Artin braid group. $B_{n}$ is presented by the braiding generators $\sigma_{1}, \ldots, \sigma_{n-1}$ and the braid relations:

$$
\begin{array}{rlll}
\sigma_{i} \sigma_{j} & =\sigma_{j} \sigma_{i} & \text { for } & |i-j|>1 \\
\sigma_{i} \sigma_{j} \sigma_{i} & =\sigma_{j} \sigma_{i} \sigma_{j} & \text { for } & |i-j|=1 .
\end{array}
$$

The framed braid group $\mathcal{F}_{n}$ is the group defined by adding to the above presentation of $B_{n}$ the framing generators $t_{1}, \ldots, t_{n}$ and the following relations:

$$
\begin{aligned}
& t_{i} t_{j}=t_{j} t_{i} \quad \text { for } \quad 1 \leq i, j \leq n \\
& t_{j} \sigma_{i}=\sigma_{i} t_{s_{i}(j)} \text { for } 1 \leq i \leq n-1 \quad \& \quad 1 \leq j \leq n \text {, }
\end{aligned}
$$

where $s_{i}(j)$ is the result of applying $s_{i}$ to $j$. The $d$-modular framed braid group, denoted $\mathcal{F}_{d, n}$, is defined by adding to the above presentation of $\mathcal{F}_{n}$ the relations:

$$
t_{i}^{d}=1 \text { for } 1 \leq i \leq n .
$$

We denote $C$ the infinite cyclic group and by $C_{d}$ the cyclic group of order $d$. We have $C \cong \mathbb{Z}$ and $C_{d} \cong \mathbb{Z} / d \mathbb{Z}$. Further, if $t$ is a generator of $C$, the group $C_{d}$ can be presented as $C_{d}=\left\langle t ; t^{d}=1\right\rangle$. From the above we have: $\mathcal{F}_{n}=C^{n} \rtimes B_{n}$ and $\mathcal{F}_{d, n}=C_{d}^{n} \rtimes B_{n}$. Finally, we shall denote the group:

$$
C_{d, n}:=C_{d}^{n} \rtimes S_{n} .
$$


1.3. From now on we fix a non-zero complex number $u$ and a positive integer $d$.

Note 1. One of the authors does not agree with the denomination 'Juyumaya trace'and the other author does not agree with the denomination 'Lambropoulou invariants'.

\section{The framization of the Hecke algebra of type $A$}

2.1. The Iwahori-Hecke algebra of type $A, \mathrm{H}_{n}(u)$, is the algebra with $\mathbb{C}$-linear basis $\left\{h_{w} \mid w \in S_{n}\right\}$ and the following rules of multiplication:

$$
h_{s_{i}} h_{w}= \begin{cases}h_{s_{i} w} & \text { for } \ell\left(s_{i} w\right)>\ell(w) \\ u h_{s_{i} w}+(u-1) h_{w} & \text { for } \ell\left(s_{i} w\right)<\ell(w)\end{cases}
$$

where $\ell$ is the usual length function on the symmetric group. Set $h_{i}:=h_{s_{i}}$. As usual we consider the presentation of the algebra $\mathrm{H}_{n}(u)$ by 'braiding' generators $h_{1}, \ldots, h_{n-1}$, subject to the braid relations (1.1) together with the quadratic relations:

$$
h_{i}^{2}=(u-1) h_{i}+u .
$$

Note that $\mathrm{H}_{n}(1)$ coincides with the group algebra $\mathbb{C} S_{n}$. The above presentation of $\mathrm{H}_{n}(u)$ says that the mapping $\pi: \sigma_{i} \mapsto h_{i}$ defines an epimorphism from $\mathbb{C} B_{n}$ onto $\mathrm{H}_{n}(u)$ whose kernel is generated by the expressions: $\sigma_{i}^{2}-(u-1) \sigma_{i}-u$.

2.2. The Hecke algebra $\mathrm{H}_{n}(u)$ is the knot algebra used to define the 2-variable Jones or Homflypt polynomial according to the Jones construction [Jo]. Namely, one uses the Markov braid equivalence on $\cup_{n} B_{n}$, comprising conjugation in the groups $B_{n}$ and positive and negative stabilization and destabilization $\left(\alpha \sigma_{n} \sim \alpha \sim \alpha \sigma_{n}^{-1} ; \alpha \in B_{n}\right)$, the map $\pi$ from $B_{n}$ to $\mathrm{H}_{n}(u)$ and the Ocneanu trace defined on $\mathrm{H}_{n}(u)$ :

Theorem 1 (Theorem $5.1[\mathrm{Jo}]$ ). Let $\zeta$ be an indeterminate over $\mathbb{C}$. Then, there exists a unique $\mathbb{C}$-linear map $\tau$ from the inductive limit of the family $\left\{\mathrm{H}_{n}(u)\right\}_{n}$ to $\mathbb{C}(\zeta)$, such that $\tau(1)=1$ and satisfying the following rules for all $a, b \in \mathrm{H}_{n}(u)$ :

$$
\begin{aligned}
\tau(a b) & =\tau(b a) \\
\tau\left(a h_{n}\right) & =\zeta \tau(a) \quad \text { (Markov property). }
\end{aligned}
$$

Diagrammatically, the Markov property of $\tau$ is illustrated on the left-hand side of Figure 3 .

Then $\tau$ has to be normalized, so that the closed braids $\widehat{\alpha}$ and $\widehat{\alpha \sigma_{n}}\left(\alpha \in B_{n}\right)$ be assigned the same value of the invariant, and also re-scaled, so that the closed braids $\widehat{\alpha \sigma_{n}^{-1}}$ and $\widehat{\alpha \sigma_{n}}$ get also assigned the same value of the invariant. So, defining:

$$
\lambda:=\frac{1-u+\zeta}{u \zeta} \quad \text { and } \quad C:=\frac{1}{\zeta \sqrt{\lambda}}
$$

then the Homflypt polynomial $P=P(u, \lambda)$ of an oriented link $L$ is defined as follows

$$
P(\widehat{\alpha})=C^{n-1}(\tau \circ \pi)(\alpha)
$$

where $\alpha$ is a braid on $n$ strands whose closure $\widehat{\alpha}$ is isotopic to $L$.

2.3. In group theory the Hecke algebra has a natural generalization, the Yokonuma-Hecke algebra $\mathrm{Y}_{d, n}(u)$, see [Yo. In J Ju1, JK] a new presentation was found for this algebra. Subsequently, in JJu2 this new presentation was considered for the Yokonuma-Hecke algebra and was proved that it supports a Markov trace. We define $\mathrm{Y}_{d, n}(u)$ as follows. 
Definition 1. The Yokonuma-Hecke algebra of type $A, \mathrm{Y}_{d, n}(u)$, is the algebra defined by the braiding generators $g_{1}, \ldots, g_{n-1}$, the framing generators $t_{1}, \ldots, t_{n}$ and the relations:

$$
\begin{aligned}
& g_{i} g_{j}=g_{j} g_{i} \quad \text { for } \quad|i-j|>1 \\
& g_{i} g_{j} g_{i}=g_{j} g_{i} g_{j} \quad \text { for } \quad|i-j|=1 \\
& t_{i} t_{j}=t_{j} t_{i} \quad \text { for } \quad 1 \leq i, j \leq n \\
& t_{j} g_{i}=g_{i} t_{s_{i}(j)} \text { for } 1 \leq i \leq n-1 \quad \& \quad 1 \leq j \leq n \\
& t_{i}^{d}=1 \quad \text { for } \quad 1 \leq i \leq n
\end{aligned}
$$

where $s_{i}(j)$ denotes the result of applying $s_{i}$ to $j$, together with the following quadratic relations:

$$
g_{i}^{2}=1+(u-1) e_{i}+(u-1) e_{i} g_{i} \quad \text { for all } \quad 1 \leq i \leq n-1
$$

where

$$
e_{i}:=\frac{1}{d} \sum_{s=0}^{d-1} t_{i}^{s} t_{i+1}^{d-s} .
$$

The elements $e_{i}$ are easily seen to be idempotents. Using this fact it follows from the quadratic relations (2.3) that the generators $g_{i}$ are invertible:

$$
g_{i}^{-1}=g_{i}+\left(u^{-1}-1\right) e_{i}+\left(u^{-1}-1\right) e_{i} g_{i} .
$$

From the above presentation it is clear that the algebra $\mathrm{Y}_{d, n}(u)$ is a quotient of the modular framed braid group algebra $\mathbb{C F}_{d, n}$ under the quadratic relations (2.3). This observation leads to diagrammatic interpretations for the elements in $\mathrm{Y}_{d, n}(u)$. For example, the elements $e_{i}$ (which are in $\mathbb{C F}_{d, n}$ and in $\left.\mathrm{Y}_{d, n}(u)\right)$ can be represented as in Figure 1.

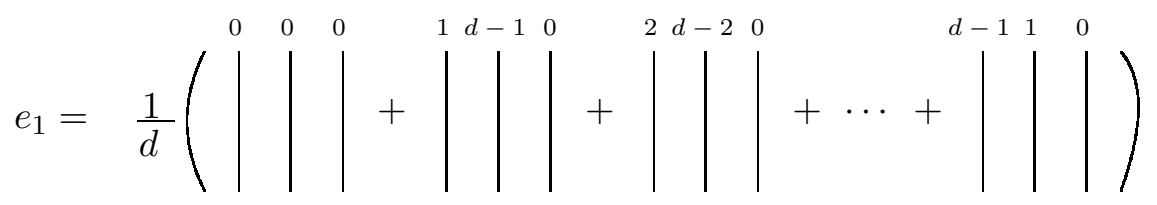

Figure 1 . The element $e_{1} \in \mathbb{C} \mathcal{F}_{d, 3}$.

Also, Eq. 2.5, which is equivalent to the quadratic relation, is illustrated in Figure 2,

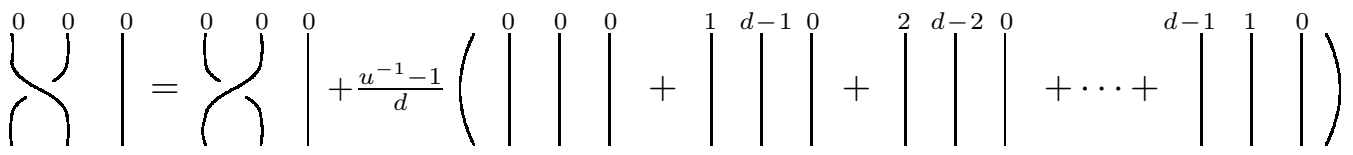

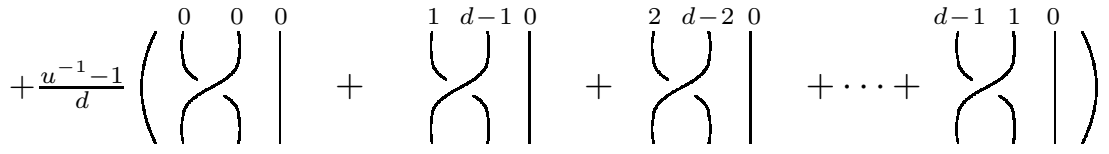

Figure 2. The element $g_{1}^{-1} \in \mathrm{Y}_{d, 3}(u)$. 
2.4. A consequence of the above definition is that every word in the defining generators of $\mathrm{Y}_{d, n}(u)$ can be written in the split form $t_{1}^{a_{1}} \ldots t_{n}^{a_{n}} g$, where the $a_{i}$ 's are integers modulo $d$ and $g$ is a word in the $g_{i}$ 's. Since the $g_{i}$ 's satisfy the braid relations we have that, if $w=s_{i_{1}} \ldots s_{i_{m}} \in S_{n}$ is a reduced expression, then the following element $g_{w}:=g_{i_{1}} \ldots g_{i_{m}}$ is well-defined. In [Ju1] it is proved that the multiplication rules in $\mathrm{Y}_{d, n}(u)$ are governed by the group $C_{d, n}$. In fact, the multiplication rules among the framing generators and between the framing generators and the braiding generators are the same multiplication rules as in the group $C_{d, n}$. For the multiplication among the braiding generators we have:

$$
g_{i} g_{w}= \begin{cases}g_{s_{i} w} & \text { for } \ell\left(s_{i} w\right)>\ell(w) \\ g_{s_{i} w}+(u-1) e_{i} g_{s_{i} w}+(u-1) e_{i} g_{w} & \text { for } \ell\left(s_{i} w\right)<\ell(w)\end{cases}
$$

Notice now that $\mathrm{Y}_{d, n}(1)=\mathbb{C} C_{d, n}$. This says that $\mathrm{Y}_{d, n}(u)$ is essentially obtained from $\mathrm{H}_{n}(u)$ by adding framing generators, since $\mathrm{H}_{n}(1)=\mathbb{C} S_{n}$. For this reason we shall call the Yokonuma-Hecke algebra $\mathrm{Y}_{d, n}(u)$ a framization of the Iwahori-Hecke algebra $\mathrm{H}_{n}(u)$. The key point in this framization is the quadratic relation (2.3), which is considered as the framization of the Hecke algebra quadratic relation.

The representation theory of the Yokonuma-Hecke algebra has been studied in [Th] and [C-PA1]. Finally, it is worth mentioning that the notions of the modular framed braid group $\mathcal{F}_{d, n}$ as well as of the Yokonuma-Hecke algebra $\mathrm{Y}_{d, n}(u)$ have been extended to constructions on the $p$-adic level [JL1, JL2] and the adelic level [JL4].

2.5. Another crucial property of the Yokonuma-Hecke algebra is that is supports a Markov trace:

Theorem 2 (Theorem 12 [Ju2]). Let $z, x_{1}, \ldots, x_{d-1}$ be indeterminates over $\mathbb{C}$. Then, there exists a unique $\mathbb{C}$-linear map tr from the inductive limit of the family $\left\{\mathrm{Y}_{d, n}(u)\right\}_{n}$ to $\mathbb{C}\left(z, x_{1}, \ldots, x_{d-1}\right)$, such that $\operatorname{tr}(1)=1$ and satisfying the following rules for all $a, b \in \mathrm{Y}_{d, n}(u)$ :

$(2)=\operatorname{tr}(a g n)=2 \operatorname{tr}(a)$

(3) $\operatorname{tr}\left(a t_{n+1}^{m}\right)=x_{m} \operatorname{tr}(a)$

(Markov property)

$(1 \leq m \leq d-1)$.

The topological interpretations for rules (2) and (3) are given in Figure 3 ,

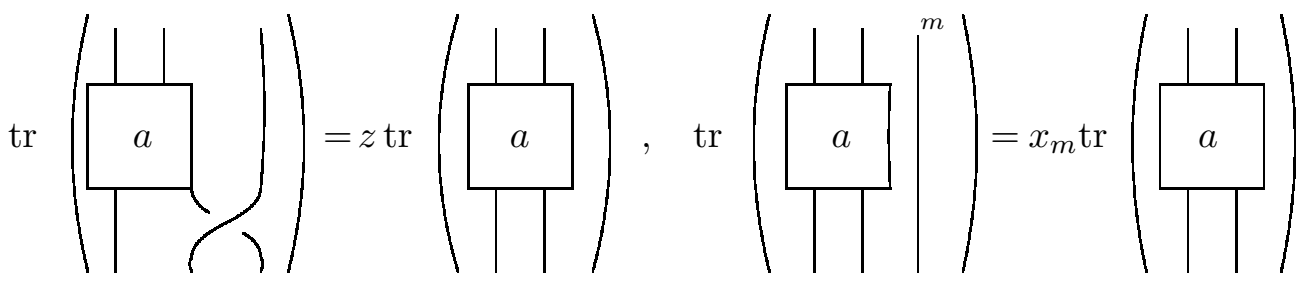

FiguRE 3. Topological interpretations of the trace rules

The trace $\operatorname{tr}$ shall be called the Juyumaya trace. Note that for $d=1$, the algebra $\mathrm{Y}_{1, n}(u)$ is the Iwahori-Hecke algebra $\mathrm{H}_{n}(u)$ and the Juyumaya trace coincides with the Ocneanu trace with parameter $z$. Note also that the trace tr lifts to the $p$-adic level [JL1, JL5] and to the adelic level [JL4].

2.6. A Markov trace is a key ingredient for constructing knot invariants from an algebra. The first key requirement is that there is a representation of some braid category to the algebra. Another key requirement is to have a Markov-type braid equivalence for the braid category, corresponding to isotopy in the related knot category. The last key ingredient is to re-scale and normalize the trace according to the given braid equivalence. 
In the case of the Yokonuma-Hecke algebras we have a natural connection with the framed braid category and with the classical braid category. More precisely, the defining relations of $\mathrm{Y}_{d, n}(u)$ yield two natural representations. One of the framed braid group:

$$
\begin{aligned}
\gamma: \mathbb{C F}_{n} & \longrightarrow \mathrm{Y}_{d, n}(u) \\
\sigma_{i} & \mapsto g_{i} \\
t_{j}^{s} & \mapsto t_{j}^{s(\bmod d)}
\end{aligned}
$$

and another of the classical braid group:

$$
\begin{aligned}
\delta: \mathbb{C} B_{n} & \longrightarrow \mathrm{Y}_{d, n}(u) \\
\sigma_{i} & \mapsto g_{i}
\end{aligned}
$$

The map $\delta$ can be viewed as the composition of the map $\gamma$ with the natural injection of $B_{n}$ into $\mathcal{F}_{n}$, whereby $\sigma_{i} \mapsto \sigma_{i}$, and classical braids are considered to have all framings zero.

In the sequel we will sometimes identify a braid $\alpha$ with its image through $\gamma$ or $\delta$.

\section{THE E-SYSTEM}

The framed braid equivalence comprises conjugation in the groups $\mathcal{F}_{n}$ and positive and negative stabilization and destabilization (see for example [KS]). So, with the trace tr in hand, in [JL5] the authors tried to obtain topological invariants for framed links after the method of V.F.R. Jones [Jo]. This meant that tr would have to be normalized, so that the closed framed braids $\widehat{\alpha}$ and $\widehat{\alpha \sigma_{n}}$ $\left(\alpha \in \mathcal{F}_{n}\right)$ get assigned the same value of the invariant, and also re-scaled, so that the closed framed braids $\widehat{\alpha \sigma_{n}^{-1}}$ and $\widehat{\alpha \sigma_{n}}\left(\alpha \in \mathcal{F}_{n}\right)$ get also assigned the same value of the invariant. However, as it turned out, $\operatorname{tr}\left(\alpha g_{n}^{-1}\right)$ does not factor through $\operatorname{tr}(\alpha)$. That is, remarkably:

$$
\operatorname{tr}\left(\alpha g_{n}^{-1}\right) \neq \operatorname{tr}\left(g_{n}^{-1}\right) \operatorname{tr}(\alpha)
$$

since, from (2.5), we deduce that $\operatorname{tr}\left(\alpha g_{n}^{-1}\right)=\operatorname{tr}\left(\alpha g_{n}\right)+\left(u^{-1}-1\right) \operatorname{tr}\left(\alpha e_{n}\right)+\left(u^{-1}-1\right) \operatorname{tr}\left(\alpha e_{n} g_{n}\right)$ and, although $\operatorname{tr}\left(\alpha e_{n} g_{n}\right)=z \operatorname{tr}(\alpha)$, the term $\operatorname{tr}\left(\alpha e_{n}\right)$ does not factor through $\operatorname{tr}(\alpha)$. Forcing the E-condition:

$$
\operatorname{tr}\left(\alpha e_{n}\right)=\operatorname{tr}\left(e_{n}\right) \operatorname{tr}(\alpha) \quad\left(a \in \mathrm{Y}_{d, n}(u)\right)
$$

implies equivalently that the trace parameters $x_{1}, \ldots, x_{d-1}$ have to satisfy the E-system [JL5], the following non-linear system of equations in $\mathbb{C}$ :

$$
E^{(m)}=x_{m} E \quad(1 \leq m \leq d-1)
$$

where

$$
E=E^{(0)}:=\operatorname{tr}\left(e_{i}\right)=\frac{1}{d} \sum_{s=0}^{d-1} x_{s} x_{d-s} \quad \text { and } \quad E^{(m)}:=\frac{1}{d} \sum_{s=0}^{d-1} x_{m+s} x_{d-s},
$$

where the sub-indices on the $x_{j}$ 's are regarded modulo $d$ and $x_{0}:=1$.

As it was shown by P. Gérardin ([JL5, Appendix]), the solutions of the E-system are parametrized by the non-empty subsets of $\mathbb{Z} / d \mathbb{Z}$.

It is worth noting that the solutions of the E-system can be interpreted as a generalization of the Ramanujan sum. Indeed, by taking the subset $R$ of $\mathbb{Z} / d \mathbb{Z}$ comprising the numbers coprime to $d$, then the solution parametrized by $R$ is, up to the factor $|R|$, the Ramanujan sum $c_{d}(k)$ (see [Ra] $)$.

It is also worth mentioning that solutions of the E-system lift to solutions on the $p$-adic level [JL1, JL5] and on the adelic level [JL4]. 


\section{Knot invariants from the Yokonuma-Hecke Algebras}

The Yokonuma-Hecke algebras have been used for constructing invariants for framed knots [JL5], for classical knots [JL4 and for singular knots [JL3. These invariants qualify the algebra $\mathrm{Y}_{d, n}(u)$ as a knot algebra and they comprise our main motivation for extending the notion of framization to other known knot algebras. We shall now recall briefly the construction of these invariants.

4.1. Let $X_{D}=\left(\mathrm{x}_{1}, \ldots, \mathrm{x}_{d-1}\right)$ be a solution of the E-system parametrized by the non-empty subset $D$ of $\mathbb{Z} / d \mathbb{Z}$.

Definition 2 (Definition $3\left[\mathrm{CL}\right.$ ). The trace map $\operatorname{tr}_{D}$ defined as the trace $\operatorname{tr}$ with the parameters $x_{i}$ specialized to the values $\mathrm{x}_{i}$, shall be called the specialized Juyumaya trace with parameter $z$.

Note that for $d=1$ the $\operatorname{trace} \operatorname{tr}_{D}$ coincides with the trace $\operatorname{tr}$ (and with the Ocneanu trace with parameter $z$ ). As it turns out [JL4, JL5],

$$
E_{D}:=\operatorname{tr}_{D}\left(e_{i}\right)=\frac{1}{|D|}
$$

where $|D|$ is the cardinal of the subset $D$.

4.2. Let $\mathcal{L}_{f}$ denote the set of oriented framed links. From the above, and re-scaling $g_{i}$ to $\sqrt{\lambda_{D}} g_{i}$, so that $\operatorname{tr}_{D}\left(g_{i}^{-1}\right)=\lambda_{D} z$, where:

$$
\lambda_{D}:=\frac{z+(1-u) E_{D}}{u z}=\frac{|D| z+1-u}{|D| u z}
$$

we have the following (mapping $\sigma_{i} \mapsto \sqrt{\lambda_{D}} g_{i}$ ):

Theorem 3 ([JL5]). Given a solution $X_{D}$ of the E-system, for any framed braid $\alpha \in \mathcal{F}_{n}$ we define for the framed link $\widehat{\alpha} \in \mathcal{L}_{f}$ :

$$
\Gamma_{D}(\widehat{\alpha})=P_{D}^{n-1}\left(\sqrt{\lambda_{D}}\right)^{\epsilon(\alpha)}\left(\operatorname{tr}_{D} \circ \gamma\right)(\alpha)
$$

where $P_{D}=\frac{1}{z \sqrt{\lambda_{D}}}, \epsilon(\alpha)$ is the algebraic sum of the exponents of the $\sigma_{i}$ 's in $\alpha$ and $\gamma$ the epimorphism (2.6). Then the map $\Gamma_{D}\left(u, \lambda_{D}\right)$ is a 2-variable isotopy invariant of oriented framed links.

Further, in JL5] a skein relation has been found for the invariant $\Gamma_{D}\left(u, \lambda_{D}\right)$ involving the braiding and the framing generators:

$$
\sqrt{\lambda} \Gamma_{D}\left(L_{-}\right)=\frac{1}{\sqrt{\lambda}} \Gamma_{D}\left(L_{+}\right)+\frac{u^{-1}-1}{d} \sum_{s=0}^{d-1} \Gamma_{D}\left(L_{s}\right)+\frac{u^{-1}-1}{d \sqrt{\lambda}} \sum_{s=0}^{d-1} \Gamma_{D}\left(L_{s \times}\right)
$$

where $L_{+}, L_{-}, L_{s}$ and $L_{s \times}, s=0, \ldots, d-1$, are diagrams of oriented framed links, which are all identical except near one crossing, where they differ by the ways indicated in Figure 4.

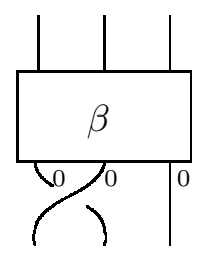

$L_{+}=\widehat{\beta \sigma_{1}}$

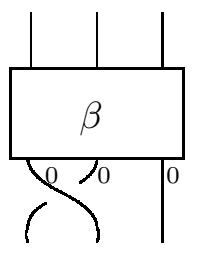

$L_{-}=\widehat{\beta \sigma_{1}^{-1}}$

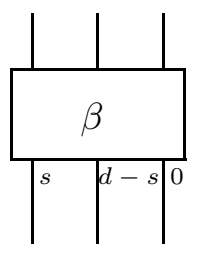

$L_{s}=\widehat{\beta t_{1}^{s} t_{2}^{d-s}}$

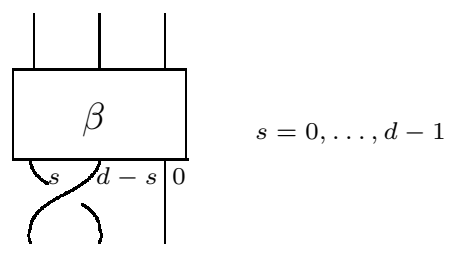

$L_{s \times}=\widehat{\beta t_{1}^{s} t_{2}^{d-s}} \sigma_{1}$

Figure 4. The framed links $L_{+}, L_{-}, L_{s}$ and $L_{s \times}$.

Finally, a $p$-adic (resp. adelic) invariant for oriented framed links, $\Gamma_{p} \infty$, has been defined through the invariants $\Gamma_{D}$. For more details see [JL5, JL4]. 
Note 2. It is logical that one should try to extract from the invariants for framed links invariants of 3-manifolds. But for this, one needs to start with invariants of unoriented framed links. This can be achieved from our constructions so far by taking appropriate quotients of the Yokonuma-Hecke algebras, analogous to the Temperley-Lieb algebras. This is presented in Section 5 .

4.3. Let $\mathcal{L}$ denote the set of oriented classical links. A classical link may be viewed as a framed link with all framings zero. By the mapping (2.7) of the classical braid group $B_{n}$ in $\mathrm{Y}_{d, n}(u)$, by the classical Markov braid equivalence and using the construction and notations above we obtain invariants for classical links, where the $t_{j}$ 's are treated as formal generators [JL4]. Namely:

Theorem 4 ([JL4]). Given a solution $X_{D}$ of the E-system, for any braid $\alpha \in B_{n}$ we define for the link $\widehat{\alpha} \in \mathcal{L}:$

$$
\Delta_{D}(\widehat{\alpha})=P_{D}^{n-1}\left(\sqrt{\lambda_{D}}\right)^{\epsilon(\alpha)}\left(\operatorname{tr}_{D} \circ \delta\right)(\alpha)
$$

where $P_{D}, \lambda_{D}$ are as defined in 4.2, $\epsilon(\alpha)$ is the algebraic sum of the exponents of the $\sigma_{i}$ 's in $\alpha$ and $\delta$ is the natural algebra homomorphism (2.7). Then the map $\Delta_{D}\left(u, \lambda_{D}\right)$ is a 2-variable isotopy invariant of oriented links.

The invariant $\Delta_{D}\left(u, \lambda_{D}\right)$ can be viewed as the restriction of $\Gamma_{D}\left(u, \lambda_{D}\right)$ on the set of framed links with all framings zero.

The invariants $\Delta_{D}\left(u, \lambda_{D}\right)$ need to be compared with known invariants of classical links, especially with the Homflypt polynomial $P(u, \lambda)$, recall $\$ 2.2$. This is not easy to do on the algebraic level as there are no algebra homomorphisms connecting the algebras and the traces [CL]. Further, the skein relation of the invariant $\Delta_{D}\left(u, \lambda_{D}\right)$ has no topological interpretation in the case of classical links. This makes the comparison very difficult also using diagrammatic methods. It is worth noting at this point that in $\mathrm{Y}_{d, n}(u)$ a 'closed' cubic relation is satisfied [JL2, JL4], closed in the sense of involving only the braiding generators, which is of minimal degree. Namely:

$$
g_{i}^{3}=u g_{i}^{2}+g_{i}-u \text {. }
$$

The cubic relation gives rise to a cubic skein relation for the invariant $\Delta_{D}\left(u, \lambda_{D}\right)$, involving only the braiding generators [JL4]:

$$
\sqrt{\lambda_{D}} \Delta_{D}\left(L_{-}\right)=-\frac{1}{u \lambda_{D}} \Delta_{D}\left(L_{++}\right)+\frac{1}{\sqrt{\lambda_{D}}} \Delta_{D}\left(L_{+}\right)+\frac{1}{u} \Delta_{D}\left(L_{0}\right)
$$

where the links $L_{++}, L_{+}, L_{0}$ and $L_{-}$have identical diagrams, except for a region where they differ in the manners illustrated in Figure 5. However, the above skein relation is not sufficient for determining the invariant $\Delta_{D}(u, z)$ diagrammatically with a simple set of initial conditions.
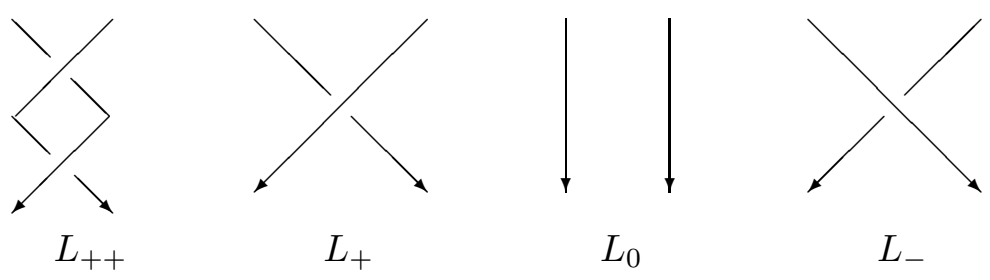

Figure 5. The classical links $L_{++}, L_{+}, L_{0}$ and $L_{-}$.

As it turns out, the cubic relation factors to the quadratic relation of the Iwahori-Hecke algebra $\mathrm{H}_{n}(u)$ :

$$
g_{i}^{3}-u g_{i}^{2}-g_{i}+u=\left(g_{i}-1\right)\left(g_{i}^{2}-(u-1) g_{i}-u\right) .
$$

Unfortunately, this factoring does not give information about the comparison of the invariants. 
From our construction it follows that we have, at least, recovered the Homflypt polynomial. In CL] it is shown that for generic values of the parameters $u, z$ the invariants $\Delta_{D}\left(u, \lambda_{D}\right)$ do not coincide with the Homflypt polynomial except in the trivial cases $u=1$ or $E=1$. More precisely, for $E=1$ an algebra homomorphism $h: \mathrm{Y}_{d, n}(u) \longrightarrow \mathrm{H}_{n}(u)$ can be defined and the composition $\tau \circ h$ is a Markov trace on $\mathrm{Y}_{d, n}(u)$ which takes the same values as the specialized Juyumaya trace $\operatorname{tr}_{D}$ whereby the $x_{i}$ 's are specialized to $d^{\text {th }}$ roots of unity [JL5, Appendix]. In this case we also obtain $|D|=1$. For details see [CL, $\S 3]$.

Yet, computational data CJJKL indicate that these invariants do not distinguish more knot pairs than the Homflypt polynomial, so they are probably topologically equivalent to the Homflypt polynomial. A complete answer to this question is still under investigation. In CJJKL some conjectures are formulated and tackled in this direction. Also, it is proved that the specialized Juyumaya trace can be computed for classical braids with rule (3) replaced by another rule involving the elements $e_{i}$.

4.4. Let $\mathcal{L}_{\mathcal{S}}$ denote the set of oriented singular links. Oriented singular links are represented by singular braids, which form the singular braid monoids $\mathcal{S} B_{n}[\mathrm{Ba}, \mathrm{Bi}, \mathrm{Sm}] . \mathcal{S} B_{n}$ is generated by the classical braiding generators $\sigma_{i}$ with their inverses and by the elementary singular braids $\tau_{i}$ which are not invertible. In [JL3] the following monoid homomorphism was constructed:

$$
\begin{aligned}
\eta: \mathcal{S} B_{n} & \longrightarrow \mathrm{Y}_{d, n}(u) \\
\sigma_{i} & \mapsto g_{i} \\
\tau_{i} & \mapsto p_{i}=e_{i}\left(1+g_{i}\right)
\end{aligned}
$$

In view of the elements $p_{i}$ the quadratic relations (2.3) may be rewritten as: $g_{i}^{2}=1+(u-1) p_{i}$. Using now the singular braid equivalence [Ge], the map $\eta$ and the specialized Juyumaya $\operatorname{trace}_{D} \operatorname{tr}_{D}$ we obtain isotopy invariants for oriented singular links [JL3]:

Theorem 5 ([JL3]). Given a solution $X_{D}$ of the E-system, for any oriented singular link $\widehat{\alpha} \in \mathcal{L}_{\mathcal{S}}$, where $\alpha \in \mathcal{S} B_{n}$, we define:

$$
H_{D}(\widehat{\alpha})=P_{D}^{n-1}\left(\sqrt{\lambda_{D}}\right)^{\epsilon(\alpha)}\left(\operatorname{tr}_{D} \circ \eta\right)(\alpha)
$$

where $P_{D}, \lambda_{D}$ are as defined in \$4.2, $\eta$ as defined in (4.6) and where $\epsilon(\alpha)$ is defined as follows: Let $\alpha=\mu_{1}^{\epsilon_{1}} \mu_{2}^{\epsilon_{2}} \ldots \mu_{m}^{\epsilon_{m}}$ where $\mu_{j} \in\left\{\sigma_{i}, \tau_{i} ; 1 \leq i \leq n-1\right\}$. Then $\epsilon(\alpha):=\epsilon_{1}+\ldots+\epsilon_{m}$, where $\epsilon_{j}=+1$ or -1 if $\mu_{j}=\sigma_{j}$ and $\epsilon_{j}=+1$ if $\mu_{j}=\tau_{j}$. Then the map $H_{D}\left(u, \lambda_{D}\right)$ is a 2-variable isotopy invariant of oriented singular links.

Moreover, in the image $\eta\left(\mathcal{S} B_{n}\right)$ the following relations hold:

$$
g_{i}^{-1}-g_{i}=\left(u^{-1}-1\right) p_{i}
$$

which give rise to the following skein relation:

$$
\sqrt{\lambda_{D}} H_{D}\left(L_{-}\right)-\frac{1}{\sqrt{\lambda_{D}}} H_{D}\left(L_{+}\right)=\frac{u^{-1}-1}{\sqrt{\lambda_{D}}} H_{D}\left(L_{\times}\right)
$$

where $L_{+}, L_{-}$and $L_{\times}$are diagrams of three oriented singular links, which are identical except near one crossing, where they are as depicted in Figure 6 .

For further details the reader is referred to JL3. We note that there are some differences from [JL3] in the signs in (4.6), (4.7) and (4.8); this is due to the change of sign in the quadratic relation (2.3). 


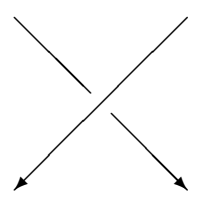

$L_{+}$

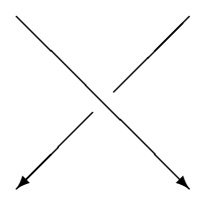

$L_{-}$

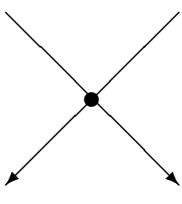

$L_{\times}$

Figure 6. The singular links $L_{+}, L_{-}$and $L_{\times}$.

4.5. Another category of knots which seems to be naturally related to the Yokonuma-Hecke algebras is the category of transverse knots, for the following reasons: transverse knots are naturally framed and oriented, and their equivalence is more refined than framed knot isotopy. In 1983 D. Bennequin $\mathrm{Be}$ noted that the closed braid presentation of knots is convenient for describing transverse knots. Further, S. Orevkov and V. Shevchishin [OS] and independently N. Wrinkle $\mathrm{Wr}$ gave a transverse analogue of the Markov theorem, comprising conjugation in the framed braid groups and positive only stabilizations and destabilizations: $\alpha \sim \alpha \sigma_{n} \in \mathcal{F}_{n+1}$, where $\alpha \in \mathcal{F}_{n}$.

Now, rule (2) of the trace $\operatorname{tr}$ (Theorem 2) tells us that it respects positive stabilizations but property (3.1) tells us that tr does not behave well under negative stabilizations. Using the above, an invariant $M\left(u, \lambda, x_{1}, \ldots, x_{d-1}\right)$ for transverse knots has been constructed in [CJJKL, which coincides with the invariant $\Gamma_{D}\left(u, \lambda_{D}\right)$ of framed knots when $\left(x_{1}, \ldots, x_{d-1}\right)$ specializes to a solution of the E-system. However, as it turns out, the invariants $M\left(u, \lambda, x_{1}, \ldots, x_{d-1}\right)$ are only topological invariants of framed knots. For details see [CJJKL].

4.6. Due to the quadratic relation (2.3) it is very difficult to do computations in the YokonumaHecke algebras and the trace tr. In http://www.math.ntua.gr/ ${ }^{\sim}$ sofia/yokonuma/index.html the reader can find a program by Sergei Chmutov and Konstantinos Karvounis, together with instructions for using it.

\section{Framization of the Temperley-Lieb algebra}

In this section we define possible framizations for the Temperley-Lieb algebra and we indicate one of them as our favourite. The results of this section are contained in GJKL1] and GJKL2].

5.1. The Temperley-Lieb algebra can be defined in several ways. Here, it is convenient to define the Temperley-Lieb algebra $\mathrm{TL}_{n}(u)$ as the quotient of the Hecke algebra $\mathrm{H}_{n}(u)$ by the two-sided ideal generated by the Steinberg elements [Jo]:

$$
h_{i, i+1}:=h_{i} h_{i+1} h_{i}+h_{i+1} h_{i}+h_{i} h_{i+1}+h_{i}+h_{i+1}+1,
$$

that is:

$$
\mathrm{TL}_{n}(u)=\frac{\mathrm{H}_{n}(u)}{\left\langle h_{i, i+1} ; \text { all } i\right\rangle} .
$$

V.F.R. Jones recovered his Markov trace on the Temperley-Lieb algebra from the Ocneanu trace $\tau[\mathrm{Jo}$ as follows: He required first that $\tau$ is zero on the Steinberg elements. This yielded two values for the parameter $\zeta$, namely:

$$
\zeta=-\frac{1}{u+1}, \quad \text { where } u \neq-1 \quad \text { and } \quad \zeta=-1 .
$$

He then showed that for these values $\tau$ is zero on all elements of the defining ideal of $\operatorname{TL}_{n}(u)$. 
Finally, the Jones polynomial $V(u)$ is obtained from the Homflypt polynomial (recall $\S 2.2$ ) by taking

Hence $\lambda=u$. That is,

$$
\zeta=-\frac{1}{u+1}
$$

$$
V(u)=P(u, u) .
$$

In order to define a framization of the Temperley-Lieb algebra, the most natural approach is to take an appropriate quotient of the Yokonuma-Hecke algebra, in analogy with the classical case. So, we try to define in $\mathrm{Y}_{d, n}(u)$ elements analogous to the $h_{i, i+1}$ 's as generators of a two-sided ideal. Now, having in mind that the multiplication rules of the Hecke algebra are governed by the symmetric group and those of $\mathrm{Y}_{d, n}(u)$ are governed by the group $C_{d, n}$ (recall Eq. 1.4), we have two obvious possibilities for generating elements, which give rise to two natural candidates for the framization of the Temperley-Lieb algebra.

5.2. For the first possibility we define for all $i$ the elements:

$$
g_{i, i+1}:=g_{i} g_{i+1} g_{i}+g_{i+1} g_{i}+g_{i} g_{i+1}+g_{i}+g_{i+1}+1 .
$$

Then, we have:

Definition 3 (GJKL1]). For $n \geq 3$, the algebra Yokonuma-Temperley-Lieb, denoted $\mathrm{YTL}_{d, n}(u)$, is defined as the quotient:

$$
\mathrm{YTL}_{d, n}(u):=\frac{\mathrm{Y}_{d, n}(u)}{\left\langle g_{i, i+1} ; \text { all } i\right\rangle} .
$$

In [CP the representations of $\mathrm{YTL}_{d, n}(u)$ are determined, as well as the dimension and an explicit basis. In GJKL1 $\mathrm{YTL}_{d, n}(u)$ is studied as a knot algebra. A presentation with non-invertible generators is given. Also, the necessary and sufficient conditions are established for the Juyumaya trace $\operatorname{tr}$ on $\mathrm{Y}_{d, n}(u)$ to pass to the quotient algebra $\mathrm{YTL}_{d, n}(u)$. Indeed we have:

Theorem 6 (Theorem 5 GJKL1]). The trace tr passes to the quotient $\mathrm{YTL}_{d, n}(u)$ if and only if the $x_{i}$ 's are solutions of the E-system and one of the two cases holds:

(i) For some $0 \leq m_{1} \leq d-1$ the $x_{\ell}$ 's are expressed as:

$$
x_{\ell}=\exp \left(\ell m_{1}\right) \quad(0 \leq \ell \leq d-1) .
$$

In this case the $x_{\ell}$ 's are $d^{\text {th }}$ roots of unity and $z=-\frac{1}{u+1}$ or $z=-1$.

(ii) For some $m_{1}, m_{2}$ such that $0 \leq m_{1} \neq m_{2} \leq d-1$ the $x_{\ell}$ 's are expressed as:

$$
x_{\ell}=\frac{1}{2}\left(\exp \left(\ell m_{1}\right)+\exp \left(\ell m_{2}\right)\right) \quad(0 \leq \ell \leq d-1) .
$$

In this case we have $z=-\frac{1}{2}$.

The cases where $z=-1$ or $z=-1 / 2$ are of no topological interest. The only interesting case is case $(i)$ for $z=-\frac{1}{u+1}$. In this case the $x_{\ell}$ 's are $d^{\text {th }}$ roots of unity, which is equivalent to $E=1$ and $|D|=1$ [JL5, Appendix]. By the discussion at the end of $\S 4.3$ the invariants $\Delta_{D}\left(u, \lambda_{D}\right)$ coincide with the Homflypt polynomial. Further, $z=-\frac{1}{u+1}$ implies $\lambda_{D}=u$. Hence, the invariants derived for classical, framed and singular knots are $\Gamma_{D}(u, u), \Delta_{D}(u, u)$ and $H_{D}(u, u)$ respectively, recall $₫ 4$.

Remark 1. In particular, the invariants:

$$
V_{D}(u):=\Delta_{D}(u, u)
$$

for classical knots all coincide with the Jones polynomial. From our point of view, this is the characteristic property of the algebra $\mathrm{YTL}_{d, n}(u)$. 
To recapitulate, the conditions on the trace tr are too strong, resulting in a trivial framization of the Jones polynomial. For this reason the algebra $\mathrm{YTL}_{d, n}(u)$ is discarded as a framization of $\mathrm{TL}_{n}(u)$.

5.3. For the second possibility we define for all $i$ the following elements, where the framings are now also involved.

$$
c_{i, i+1}:=\sum_{\alpha, \beta, \gamma \in \mathbb{Z} / d \mathbb{Z}} t_{i}^{\alpha} t_{i+1}^{\beta} t_{i+2}^{\gamma} g_{i, i+1},
$$

where $g_{i, i+1}$ as defined in (5.3). Then, we obtain:

Definition 4 (GJKL2]). The Complex Reflection Temperley-Lieb algebra, denoted $\mathrm{CTL}_{d, n}(u)$, is defined as the quotient:

$$
\mathrm{CTL}_{d, n}(u):=\frac{\mathrm{Y}_{d, n}(u)}{\left\langle c_{i, i+1} ; \text { all } i\right\rangle}
$$

In GJKL2 the necessary and sufficient conditions are determined for the trace tr to pass to $\mathrm{CTL}_{d, n}(u)$. More precisely, we have the following:

Theorem 7 (GJKL2]). The trace tr passes to the quotient $\mathrm{CTL}_{d, n}(u)$ if and only if the parameter $z$ and the $x_{i}$ 's are related through the equation:

$$
(u+1) z^{2} \sum_{k \in \mathbb{Z} / d \mathbb{Z}} x_{k}+(u+2) z \sum_{k \in \mathbb{Z} / d \mathbb{Z}} E^{(k)}+\sum_{k \in \mathbb{Z}} \operatorname{tr}\left(e_{1}^{(k)} e_{2}\right)=0
$$

where

$$
e_{1}^{(k)}:=\frac{1}{d} \sum_{s=0}^{d-1} t_{1}^{k+s} t_{2}^{d-s} \quad \text { and } \quad E^{(k)}:=\operatorname{tr}\left(e_{1}^{(k)}\right) \quad(0 \leq k \leq d-1)
$$

and where $e_{1}^{(0)}=e_{1}$ and $E^{(0)}=E$.

Remark 2. Contrary to the case of $\mathrm{YTL}_{d, n}(u)$, the conditions of Theorem 7 are too relaxed on the trace parameters $x_{i}$. However, in order to obtain framed, classical or singular link invariants from the algebras $\mathrm{CTL}_{d, n}(u)$ one has to impose the $\mathrm{E}$-condition on the $x_{i}$ 's. This in turn leads to the following values for $z$ (see GJKL2]):

$$
z=-\frac{1}{(u+1)|D|} \text { or } z=-\frac{1}{|D|} \text {. }
$$

Again, the interesting value is $z=-1 /(u+1)|D|$ and, as it turns out, the link invariants one obtains coincide with those from the algebras $\operatorname{FTL}_{d, n}(u)$ that we define next.

5.4. From the above constructions and from the knot algebras point of view it follows that it would be more sensible to consider an intermediate algebra between $\mathrm{CTL}_{d, n}(u)$ and $\mathrm{YTL}_{d, n}(u)$, for which the conditions for the trace tr to pass through include explicitely all solutions of the E-system. Indeed, for all $i$ we define the elements:

$$
r_{i, i+1}:=\sum_{\substack{\alpha, \beta, \gamma \in \mathbb{Z} / d \mathbb{Z} \\ \alpha+\beta+\gamma=0}} t_{i}^{\alpha} t_{i+1}^{\beta} t_{i+2}^{\gamma} g_{i, i+1}=e_{i} e_{i+1} g_{i, i+1}
$$

We now define:

Definition 5 (GJKL2]). The framization of the Temperley-Lieb algebra, denoted $\mathrm{FTL}_{d, n}(u)$, is defined as the quotient:

$$
\mathrm{FTL}_{d, n}(u):=\frac{\mathrm{Y}_{d, n}(u)}{\left\langle r_{i, i+1} ; \text { all } i\right\rangle}
$$


For the algebra $\mathrm{FTL}_{d, n}(u)$ we have determined in GJKL2 the necessary and sufficient conditions on the trace parameters $z$ and the $x_{i}$ 's for the trace tr to pass to $\mathrm{FTL}_{n}(u)$. In order to state our result we need to introduce the following notation: given a sequence $\left(x_{1}, \ldots, x_{d-1}\right)$ of $d-1$ complex numbers, we denote by $x$ the function on $\mathbb{Z} / d \mathbb{Z}$ with values in $\mathbb{C}$, such that 0 is mapped to 1 and $i$ in mapped to $x_{i}$, for $1 \leq i \leq d-1$. Further, we denote $\widehat{x}$ the Fourier transform of $x$ (cf. GJKL2, $\S 3])$.

We then have the following:

Theorem 8 (GJKL2]). The trace tr passes to $\mathrm{FTL}_{d, n}(u)$ if and only if the trace parameters satisfy

$$
x_{m}=-z\left(\sum_{k \in D_{1}} \exp (k m)+\sum_{k \in D_{2}} \exp (k m)\right) \quad \text { and } \quad z=-\frac{1}{\left|D_{1}\right|+(u+1)\left|D_{2}\right|}
$$

where the disjoint union $D_{1} \cup D_{2}$ is the support of the Fourier transform $\hat{x}$ of $x$,

$$
D_{1}:=\left\{k \in \mathbb{Z} / d \mathbb{Z} ; y_{k}=-d z\right\}, \quad D_{2}:=\left\{k \in \mathbb{Z} / d \mathbb{Z} ; y_{k}=-d z(u+1)\right\},
$$

and the $y_{k}$ 's denote the values of $\hat{x}$.

In particular, the above theorem implies that whenever the trace parameters are solutions of the E-system, then the trace tr passes to $\mathrm{FTL}_{d, n}(u)$. Indeed, we have the following corollary.

Corollary 1 (GJKL2]). In the case where one of the sets $D_{1}$ or $D_{2}$ is the empty set we obtain that the values in the above theorem become solutions of the E-system. More precisely, in the case where $D_{1}$ is the empty set, the $x_{m}$ 's become the solutions of the E-system parametrized by $D_{2}$ and $z$ takes the value $z=-1 /(u+1)\left|D_{2}\right|$. In the case where $D_{2}$ is the empty set we have that the $x_{m}$ 's become the solutions of the E-system parametrized by $D_{1}$ and $z$ takes the value $z=-1 /\left|D_{1}\right|$.

The above Corollary allows one to define a non-trivial version of a framed Jones polynomial in the same manner as the Jones polynomial coincides with $P(u, u)$, the specialization of the Homflypt polynomial $P(u, \lambda)$ for $z=-1 /(u+1)$. More precisely, we give the following definition, which is possible by Corollary 1 and Eq. 3.2 .

Definition 6 (GJKL2]). The framed Jones polynomial $\mathcal{V}_{D}(u)$ is defined as the specialization of the polynomial $\Gamma_{D}\left(u, \lambda_{D}\right)$ at the value:

$$
z=-\frac{1}{(u+1)|D|}
$$

Hence:

$$
\mathcal{V}_{D}(u):=\Gamma_{D}(u, u)
$$

Similarly, one derives the invariants $\Delta_{D}(u, u)$ and $H_{D}(u, u)$ for classical and singular links respectively from the invariants $\Delta_{D}\left(u, \lambda_{D}\right)$ and $H_{D}\left(u, \lambda_{D}\right)$ (recall 㓙) by specializing at $z=-1 /(u+1)|D|$.

Remark 3. The invariants for classical links $\Delta_{D}(u, u)$ may prove to be topologically equivalent with the Jones polynomial, in analogy with $\Delta_{D}\left(u, \lambda_{D}\right)$ and the Homflypt polynomial (recall discussion at the end of $\S 4.3$ ). In case they are not, one could compare corresponding 3-manifold invariants with the Witten invariants from the Jones polynomial, see [We].

5.5. All three quotient algebras $\operatorname{YTL}_{d, n}(u), \mathrm{FTL}_{d, n}(u)$ and $\mathrm{CTL}_{d, n}(u)$ equipped with the Markov traces are interesting on their own right. They are related via the following algebra epimorphisms:

$$
\mathrm{Y}_{d, n}(u) \rightarrow \mathrm{CTL}_{d, n}(u) \rightarrow \mathrm{FTL}_{d, n}(u) \rightarrow \mathrm{YTL}_{d, n}(u),
$$

which follow from inclusions of their defining ideals GJKL2]. 


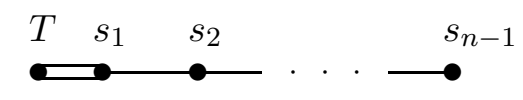

FiguRE 7. The Dynkin diagram of type $B$.

\section{Framization of the Hecke-Related algebras of type $B$}

6.1. The Artin group of type $B$, denoted $B_{1, n}$, is related to the following Dynkin diagram:

That is, $B_{1, n}$ is presented by the braiding generators $\sigma_{1}, \ldots, \sigma_{n-1}$ and the loop generator $T$ (see Figure 8), satisfying the braid relations (1.1) and the relations:

$$
\begin{aligned}
T \sigma_{1} T \sigma_{1} & =\sigma_{1} T \sigma_{1} T \\
T \sigma_{i} & =\sigma_{i} T \quad \text { for } \quad 2 \leq i \leq n-1 .
\end{aligned}
$$

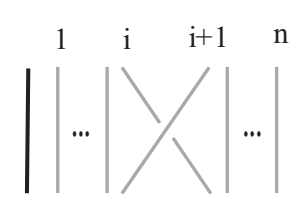

$\sigma_{\mathrm{i}}$

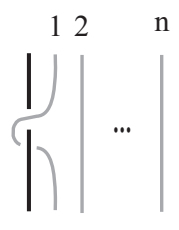

$T$

FIGURE 8. The braiding generators and the loop generator of $B_{1, n}$.

Geometrically, a braid in $B_{1, n}$ has $n+1$ strands, with the first strand identically fixed and the other $n$ strands numbered from 1 to $n$. See Figure 9 for an example. Its closure is an oriented link in the solid torus, where the complement solid torus is represented by the closure of the fixed strand [La1, La2].

Remark 4. The Artin braid group of type $B, B_{1, n}$, is isomorphic to the affine Artin braid group of type $A$, denoted $\widetilde{B_{n}}$. Thus, another geometric interpretation for elements in $B_{1, n}$ is as cylinder braids, that is, as braids in a thickened cylinder. Then, depending on how closure is defined (by simple closed arcs in the thickened cylinder or by endpoints identifications), they could give rise to oriented links in the solid torus or in the thickened torus.
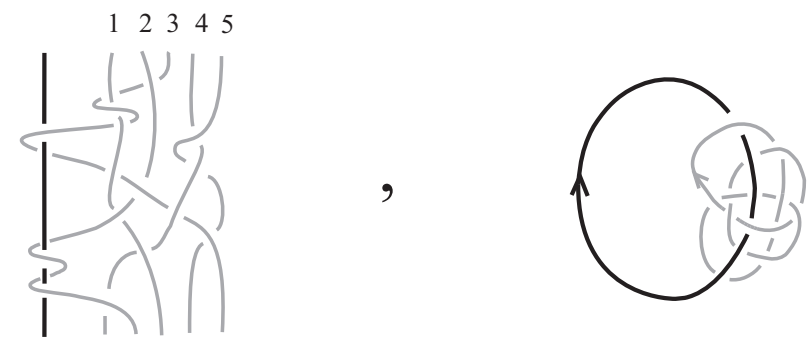

Figure 9. A braid in $B_{1, n}$ and a link in the solid torus. 
6.2. For $u, U \in \mathbb{C} \backslash\{0\}$, the classical Iwahori-Hecke algebra of type $B$ [DJ], denoted here $\mathrm{H}_{1, n}(u, U)$, can be viewed as the quotient of the group algebra $\mathbb{C} B_{1, n}$ over the expressions:

$$
\sigma_{i}^{2}-(u-1) \sigma_{i}-u \quad \text { and } \quad T^{2}-(U-1) T-U .
$$

Further, for $u, u_{1}, \ldots, u_{r} \in \mathbb{C} \backslash\{0\}$, the cyclotomic Hecke algebra of type $B$ and degree $r$ [AK, BM], denoted here $\mathrm{H}_{1, n}\left(u, u_{1}, \ldots, u_{r}\right)$, can be defined as the quotient of the group algebra $\mathbb{C} B_{1, n}$ over the expressions:

$$
\sigma_{i}^{2}-(u-1) \sigma_{i}-u \quad \text { and } \quad\left(T-u_{1}\right)\left(T-u_{2}\right) \ldots\left(T-u_{r}\right) .
$$

For $r=2$ the algebra $\mathrm{H}_{1, n}\left(u, u_{1}, u_{2}\right)$ can be proved to be isomorphic to $\mathrm{H}_{1, n}(u, U)$.

Finally, the generalized Hecke algebra of type $B$ [La2], denoted $\mathrm{H}_{1, n}(u)$, is defined as the quotient of the group algebra $\mathbb{C} B_{1, n}$ over the expressions:

$$
\sigma_{i}^{2}-(u-1) \sigma_{i}-u
$$

The algebra $\mathrm{H}_{1, n}(u)$ was observed by T. tom Dieck [La2, Remark 1] to be isomorphic to the affine Hecke algebra of type $A$.

In [La1, GL] and [La2] Markov traces have been constructed on all these algebras, giving rise to all possible analogues of the Homflypt polynomial for oriented links in the solid torus. The two rules of these traces, namely conjugation and Markov property, are analogous to the two rules of the Ocneanu trace (recall Theorem 1). Then, there is also a third inductive rule that takes care of the loopings, namely:

$$
\tau\left(a\left(h_{n} \ldots h_{1} T^{k} h_{1}^{-1} \ldots h_{n}^{-1}\right)\right)=s_{k} \tau(a),
$$

where $\sigma_{i}$ corresponds to the braiding generator $h_{i}$ and $T$ corresponds to $T$, and where $a \in \mathrm{H}_{1, n}(u, U)$ or $\mathrm{H}_{1, n}\left(u, u_{1}, \ldots, u_{r}\right)$ or $\mathrm{H}_{1, n}(u)$, depending on the algebra we are in each time. For details see [La1, GL] and [La2].

6.3. From the above it is natural to try to define framizations of the Hecke algebra of type $B$, of the cyclotomic Hecke algebra of type $B$ and of the generalized Hecke algebra of type $B$, all $B$-type analogues of the Yokonuma-Hecke algebra, with the prospective to obtain invariants of links in the solid torus, framed, classical or singular, analogous to those defined by the Yokonuma-Hecke algebra. For this we define first:

Definition 7 (Definition 4 [JL6]). The framed braid group of type $B, \mathcal{F}_{1, n}$, is the group presented by the generators $T, \sigma_{1}, \ldots, \sigma_{n-1}$ of $B_{1, n}$ together with the framing generators $t_{1}, \ldots, t_{n}$, subject to the relations of $B_{1, n}$ together with the framing relations (1.2) and the relations:

$$
t_{i} T=T t_{i} \text { for all } 1 \leq i \leq n .
$$

Geometrically, elements in $\mathcal{F}_{1, n}$ have framings on the $n$ numbered strands and upon closure they represent framed links in the solid torus. The $d$-modular framed braid group of type $B$, denoted $\mathcal{F}_{d, 1, n}$, is defined by adding to the above presentation of $\mathcal{F}_{1, n}$ the relations:

$$
t_{i}^{d}=1 \text { for } 1 \leq i \leq n .
$$

Remark 5. By Remark $4 \mathcal{F}_{1, n}$ is isomorphic to the affine framed braid group of type $A$, denoted $\widetilde{\mathcal{F}_{n}}$. So, elements in $\mathcal{F}_{1, n}$ can be also interpreted as framed braids in a thickened cylinder. Then, upon different types of closures, they give rise to oriented framed links in the solid torus or in the thickened torus. 
6.4. Define now the following elements in $\mathbb{C F}_{d, 1, n}$ :

$$
e_{i}:=\frac{1}{d} \sum_{m=0}^{d-1} t_{i}^{m} t_{i+1}^{d-m} \quad \text { for all } \quad 1 \leq i \leq n-1 .
$$

It is clear that the elements $e_{i}$ are idempotents. The $e_{i}$ 's are represented geometrically as in Figure 1, but with the addition in all terms of a first fixed strand with no framing.

We shall now define framizations of the $B$-type algebras above. These definitions have all been given in [JL6, Definition 5].

Definition 8 ([JL6]). For $u, U \in \mathbb{C} \backslash\{0\}$ we define the Yokonuma-Hecke algebra of type $B, \mathrm{Y}_{d, 1, n}(u, U)$, as the algebra presented by the braiding generators $g_{1}, \ldots, g_{n-1}$, the loop generator $T$ and the framing generators $t_{1}, \ldots, t_{n}$, subject to the relations (2.2) for the braiding and framing generators, together with the following extra relations:

$$
\begin{aligned}
T g_{1} T g_{1} & =g_{1} T g_{1} T & & \\
T g_{i} & =g_{i} T & & \text { for } \quad 2 \leq i \leq n-1 \\
T t_{j} & =t_{j} T & & \text { for } \quad 1 \leq j \leq n
\end{aligned}
$$

and the quadratic relations:

$$
g_{i}^{2}=1+(u-1) e_{i}+(u-1) e_{i} g_{i} \quad(1 \leq i \leq n-1)
$$

and

$$
T^{2}=(U-1) T+U
$$

The generators $g_{i}$ and $T$ are are easily seen to be invertible:

$$
g_{i}^{-1}=g_{i}+\left(u^{-1}-1\right) e_{i}+\left(u^{-1}-1\right) e_{i} g_{i} \quad \text { and } \quad T^{-1}=U^{-1} T+\left(U^{-1}-1\right) .
$$

From the above presentation it is clear that the algebra $\mathrm{Y}_{d, 1, n}(u, U)$ is a quotient of the modular framed braid group algebra $\mathbb{C F}_{d, 1, n}$ under the quadratic relations (6.3) and (6.4). This observation leads to diagrammatic interpretations for the elements of $\mathrm{Y}_{d, 1, n}(u, U)$.

Definition 9 ([JL6]). For $u, u_{1}, \ldots, u_{r} \in \mathbb{C} \backslash\{0\}$ we define the cyclotomic Yokonuma-Hecke algebra of type $B$ and of degree $r, \mathrm{Y}_{d, 1, n}\left(u, u_{1}, \ldots, u_{r}\right)$, as the algebra presented by the braiding generators $g_{1}, \ldots, g_{n-1}$, the loop generator $T$ and the framing generators $t_{1}, \ldots, t_{n}$ subject to the relations (2.2) for the braiding and framing generators, relations (6.2) for the loop generator, the quadratic relations (6.3) for the braiding generators and the following polynomial relation for the loop generator $T$ in place of $(6.4)$ :

$$
\left(T-u_{1}\right)\left(T-u_{2}\right) \ldots\left(T-u_{r}\right)=0 .
$$

Note that the generators $g_{i}$ and $T$ are invertible. Also, that the algebra $\mathrm{Y}_{d, 1, n}\left(u, u_{1}, \ldots, u_{r}\right)$ is clearly a quotient of the modular framed braid group algebra $\mathbb{C} \mathcal{F}_{d, 1, n}$ under the quadratic relations (6.3) and relation (6.6).

Definition 10 ([JL6]). For $u \in \mathbb{C} \backslash\{0\}$ we define the generalized Yokonuma-Hecke algebra of type $B, \mathrm{Y}_{d, 1, n}(u)$, as the algebra presented by the braiding generators $g_{1}, \ldots, g_{n-1}$, the loop generator $T$ and the framing generators $t_{1}, \ldots, t_{n}$ subject to the relations (2.2) for the braiding and framing generators, relations (6.2) for the loop generator and the quadratic relations (6.3) for the braiding generators.

Remark 6. By [La2, Remark 1] and Remark 5, the algebra $\mathrm{Y}_{d, 1, n}(u)$ can be considered isomorphically as the affine Yokonuma-Hecke algebra of type A. See also [C-PA1]. 
Note that for $d=1$ the algebras $\mathrm{Y}_{1,1, n}(u, U), \mathrm{Y}_{1,1, n}\left(u, u_{1}, \ldots, u_{r}\right)$ and $\mathrm{Y}_{1,1, n}(u)$ coincide with the corresponding algebras of $B$-type defined above. Note also that the algebras $\mathrm{Y}_{d, 1, n}(u, U)$ and $\mathrm{Y}_{d, 1, n}\left(u, u_{1}, \ldots, u_{r}\right)$ can be clearly viewed as quotients of $\mathrm{Y}_{d, 1, n}(u)$ by the relations (6.4) and (6.6) respectively. Namely, we have algebra epimorphisms:

$$
\mathbb{C} \mathcal{F}_{d, 1, n} \rightarrow \mathrm{Y}_{d, 1, n}(u) \rightarrow \mathrm{Y}_{d, 1, n}\left(u, u_{1}, \ldots, u_{r}\right) .
$$

On all these algebras unique Markov traces can be constructed with the three rules analogous to the rules of the Markov traces discussed in $\S 6.2$, together with a fourth inductive rule that takes care of the framings, analogous to rule (3) of the Juyumaya trace $\operatorname{tr}$ (recall Theorem 2). Such traces are constructed in [C-PA2]. Further, invariants of framed links, classical links and singular links in the solid torus can be constructed, up to necessary conditions analogous to the E-condition. These conditions are given in [C-PA2], where the representation theory of all these algebras is also studied.

\section{Framization of the BMW algebra}

7.1. Let $l, m \in \mathbb{C} \backslash\{0\}$. Birman and Wenzl $[\mathrm{BW}]$ and simultaneously but independently Murakami [Mu defined a unital associative algebra $C_{n}=C_{n}(l, m)$ which is known as the Birman-WenzlMurakami algebra or simply the $B M W$ algebra. The algebra $C_{n}$ is defined by two sets of generators: the 'braiding' generators $g_{1}, \ldots, g_{n-1}$ and the 'tangle' generators $h_{1}, \ldots, h_{n-1}$, satisfying: the braid relations (1.1) among the $g_{i}$ 's, together with the relations:

$$
\begin{array}{rlrl}
g_{i} h_{i} & =l^{-1} h_{i} & & \text { for } \quad 1 \leq i \leq n-1 \\
h_{i} g_{i \pm 1} h_{i} & =l h_{i} & \text { for } \quad \text { all } i \\
g_{j} h_{i} & =h_{i} g_{j} & \text { for } \quad|i-j| \geq 2
\end{array}
$$

and the quadratic relations:

$$
g_{i}^{2}=1-m g_{i}+m l^{-1} h_{i} \quad(1 \leq i \leq n-1) .
$$

For diagrammatic interpretations of the 'tangle' generators $h_{i}$ the reader may view Figure 10, where the framings 0 and 1 should be ignored and where $h_{i}$ corresponds to the two horizontal arcs joining the $i$ and $i+1$ endpoints.

From the defining relations of $C_{n}$ we deduce that the $g_{i}$ 's are invertible:

$$
g_{i}^{-1}=g_{i}-m h_{i}+m
$$

and also the following important relations:

$$
\begin{array}{rlll}
h_{i} g_{i} & =l^{-1} h_{i} & \text { for } & 1 \leq i \leq n-1 \\
h_{i} h_{j} & =h_{j} h_{i} & \text { for } & |i-j| \geq 2 \\
h_{i}^{2} & =y h_{i} & \text { for } & 1 \leq i \leq n-1
\end{array}
$$

where

$$
y:=1+\frac{l^{-1}-l}{m} .
$$

The algebra $C_{n}$ is a quotient of the classical braid group algebra $\mathbb{C} B_{n}$. To see this consider $C_{n}$ generated by the $g_{i}$ 's only, and view Eq. 7.3 as the defining relations for the $h_{i}$ 's. Further, the element $h_{i}$ can be seen represented in the category of $(n, n)$-tangles as the elementary tangle consisting in two curved parallel horizontal arcs joining the endpoints $i$ and $i+1$ at the top and at the bottom of the otherwise identity tangle (see Figure 10). The algebra $C_{n}$ is related to the Kauffman polynomial invariant for classical knots Ka1. 


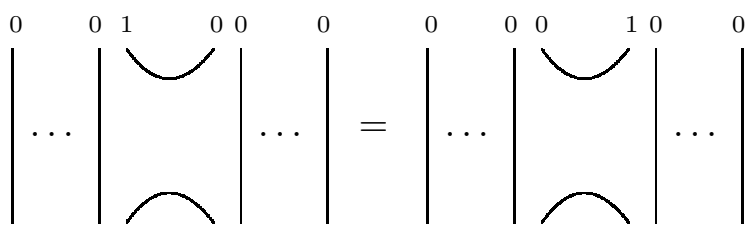

FIGURE 10. The relation $t_{i} h_{i}=t_{i+1} h_{i}$.

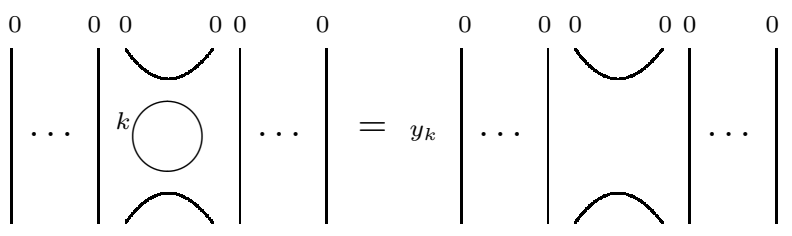

FiguRE 11. The relation $h_{i} t_{i}^{k} h_{i}=y_{k} h_{i}$.

7.2. In [JL6] the framization of the BMW algebra has been introduced. We shall decribe it here briefly.

Definition 11. Let $y_{0}:=y$ and $y_{1}, \ldots, y_{d-1}$ in $\mathbb{C} \backslash\{0,1\}$. The framization of the BMW algebra $C_{n}$, denoted $F_{d, n}=F_{d, n}\left(l, m, y_{0}, \ldots, y_{d-1}\right)$, is defined through three sets of generators: the two sets of generators of the algebra $C_{n}$ given above, together with the framing generators $t_{1}, \ldots, t_{n}$, satisfying all defining relations of $C_{n}$ except for the quadratic relations (17.2), which are replaced by the following quadratic relations:

$$
g_{i}^{2}=(1-m)-m e_{i}\left(g_{i}-1\right)+m l^{-1} h_{i} \quad(1 \leq i \leq n-1)
$$

where $e_{i}$ as defined in (2.4), together with the following relations for the framing generators:

$$
\begin{array}{rlll}
t_{i}^{d}=1 & \text { and } t_{i} t_{j}=t_{j} t_{i} & & \text { for } 1 \leq i, j \leq n \\
t_{j} g_{i} & =g_{i} t_{s_{i}(j)} & & \text { for } 1 \leq i \leq n-1 \quad \& \quad 1 \leq j \leq n \\
t_{i} h_{i} & =t_{i+1} h_{i} & & \text { for } 1 \leq i \leq n-1 \\
h_{i} t_{i} & =h_{i} t_{i+1} & & \text { for } 1 \leq i \leq n-1 \\
h_{i} t_{i}^{k} h_{i} & =y_{k} h_{i} & & \text { for } 1 \leq i \leq n-1 \quad \& \quad 0 \leq k \leq d-1 \\
h_{i} t_{j} & =t_{j} h_{i} & \text { for } \quad j \neq i, i+1
\end{array}
$$

where $s_{i}(j)$ is the effect of the transposition $s_{i}=(i, i+1)$ on $j$.

Note that for $d=1$ we have $e_{i}=1$, hence $F_{1, n}$ coincides with $C_{n}$. Also, the elements $g_{i}$ are invertible [JL6, Proposition 1]:

$$
g_{i}^{-1}=\frac{1}{1-m} g_{i}-\frac{m}{1-m} g_{i} e_{i}-m h_{i}+m e_{i} .
$$

The algebra $F_{d, n}$ can be viewed as a quotient of the modular framed braid group algebra $\mathbb{C} \mathcal{F}_{d, n}$. To see this, in analogy to the classical case we exempt the $h_{i}$ 's from the set of generators for the algebra $F_{d, n}$ and we consider Eq. 7.7 as the defining relations for the $h_{i}$ 's. So, elements in the algebra $F_{d, n}$ can be viewed as framed $(n, n)$-tangles, with framings modulo $d$. In this context, Figures 10 and 11] illustrate two of the relations of (7.6).

In [JL6] it is shown that $F_{d, n}$ is finite dimensional. We also have the following important result.

Proposition 1 (Proposition 2 [JL6]). The elements $g_{i}$ satisfy the quartic relation:

$$
g_{i}^{4}+m g_{i}^{3}+(m-2) g_{i}^{2}+m(m-1) g_{i}-(m-1)=m l^{-1}\left(m+l^{-2}-1\right) h_{i}
$$


and this is of minimal degree not containing the framing generators $t_{i}$. Also, they satisfy the 'closed' quintic relation:

$$
\left(x-l^{-1}\right)\left(x^{4}+m x^{3}+(m-2) x^{2}+m(m-1) x-(m-1)\right)=0
$$

and this is of minimal degree not containing the generators $t_{i}$ and $h_{i}$. Moreover:

$$
x^{4}+m x^{3}+(m-2) x^{2}+m(m-1) x-(m-1)=\left(x^{2}+m x-1\right)\left(x^{2}+m-1\right) .
$$

Finally we have the following result:

Proposition 2. [JL6, Proposition 3] Any element in $F_{d, n}$ can be written as a $\mathbb{C}$-linear combination of monomials of the form $\alpha f \beta$, where $\alpha$ and $\beta$ are monomials in $1, g_{1}, \ldots, g_{n-2}, h_{1}, \ldots, h_{n-2}$, $t_{1}, \ldots, t_{n-1}$ and $f \in X_{n}:=\left\{t_{n}^{s}, g_{n-1}, t_{n-1}^{s} h_{n-1} t_{n-1}^{r} \quad \mid \quad 0 \leq r, s \leq d-1\right\}$.

This result is in the right direction for defining Markov traces on $F_{d, n}$ via inductive rules.

\section{The framization of the Singular Hecke algebra}

A definition of the singular Hecke algebra, denoted $S \mathrm{H}_{n}(u)$, was proposed by Paris and Rabenda $[\mathrm{PR}$. This algebra is a complex associative unital algebra defined as the quotient of the algebra $\mathbb{C} S B_{n}$ of the singular braid monoid $S B_{n}$ over the Hecke algebra quadratic relations (2.1).

Recall that $S B_{n}$ was introduced in different contexts by Baez [Ba], Birman [Bi] and Smolin [Sm] and it is defined by the classical elementary braids $\sigma_{i}$ with their inverses $\sigma_{i}^{-1}, i=1, \ldots, n-1$, which are subject to the braid relations (1.1), and by the elementary singular braids $\tau_{i}, i=1, \ldots, n-1$, together with the following relations:

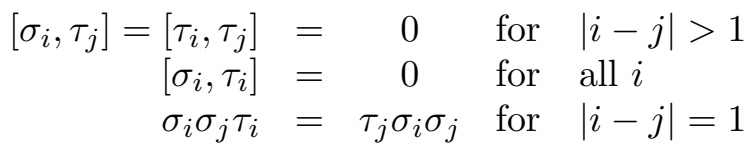

Paris and Rabenda $[\mathrm{PR}]$ constructed also a universal Markov trace on these algebras, which lead to an invariant for singular knots, which is basically equivalent to the singular link invariant of Kauffman and Vogel defined in [KV].

We can now proceed with the following definition.

Definition 12. The framization of the algebra $S \mathrm{H}_{n}(u)$, denoted $F S_{d, n}(u)$, is defined as the unital associative algebra over $\mathbb{C}$, defined through three sets of generators: $g_{1}, \ldots, g_{n-1}, \tau_{1}, \ldots, \tau_{n-1}$ corresponding to the two sets of generators of the algebra $S \mathrm{H}_{n}(u)$ given above, together with the framing generators $t_{1}, \ldots, t_{n}$, satisfying all the Yokonuma-Hecke algebra relations, (2.2) and (2.3), together with the above relations (8.1) of $S B_{n}(u)$, whereby $\sigma_{i}$ corresponds to $g_{i}$ and $\tau_{i}$ to $\tau_{i}$.

For the algebra $F S_{d, n}(u)$ one needs to find appropriate inductive basis and define on it a Markov trace analogue to the one by Paris and Rabenda.

\section{Concluding note}

We presented framizations of several knot algebras, starting from the example of the classical Iwahori-Hecke algebra, and discussed questions that need to be further investigated. There are many more other knot algebras. For example, other quotients of the classical braid group, quotients of the virtual braid group [Ka2, $\mathrm{KL}$, or the Rook algebra [BRY], which is related to the Alexander polynomial. 


\section{REFERENCES}

[AK] S. Ariki, K. Koike, A Hecke algebra of $\mathbb{Z} / r \mathbb{Z} 2 S_{n}$ and construction of its irreducible representations, Advances in Math. 106 (1994), 216-243.

[Ba] J.C. Baez, Link invariants of finite type and perturbation theory. Lett. Math. Phys. 26, No. 1 (1992), $43-51$.

[Be] D. Bennequin, Entrelacements et équations de Pfaffe, Asterisque, 107-108 (1983), 87-161.

[BRY] S. Bigelow, E. Ramos, R. Yin The Alexander and Jones Polynomials through representations of the Rook algebra, J. Knot Theory and Its Ramifications 21, No. 12 (2012).

[Bi] J.S. Birman, New points of view in knot theory, Bull. Amer. Math. Soc. (N.S.) 28, No. 2 (1993), $253-287$.

[BW] J. Birman, H. Wenzl, Braids link polynomials and a new algebra, Trans. of the Amer. Math. Soc. 313, No. 1 (1989), 249-273.

[BM] M. Broué, G. Malle, Zyklotomische Heckealgebren, Astérisque 212 (1993), 119-203.

[CL] M. Chlouveraki, S. Lambropoulou, The Yokonuma-Hecke algebras and the HOMFLYPT polynomial, J. Knot Theory and Its Ramifications 22, No. 14 (2013) 1350080 (35 pages), DOI: 10.1142/S0218216513500806.

[CP] M. Chlouveraki, G. Pouchin, Determination of the representations and a basis for the YokonumaTemperleyLieb algebra, to appear in Algebras and Representation Theory. See also arXiv:1311.5626.

[C-PA1] M. Chlouveraki, L. Poulain D'Andecy, Representation theory of the Yokonuma-Hecke algebra, Advances in Mathematics 259 (2014), 134172.

[C-PA2] M. Chlouveraki, L. Poulain D'Andecy, Markov traces on affine and cyclotomic Yokonuma-Hecke algebras. See arXiv: $1406.3207 \mathrm{v} 1$.

[CJJKL] S. Chmutov, S. Jablan, J. Juyumaya, K. Karvounis, S. Lambropoulou, On the knot invariants from the Yokonuma-Hecke algebras, in preparation. See http://www.math.ntua.gr/sofia/yokonuma/index.html.

[DJ] R. Dipper, G.D. James, Representations of Hecke algebras of type $B_{n}$, Journal of algebra 146 (1992), $454-481$.

[GL] M. Geck, S. Lambropoulou, Markov traces and knot invariants related to Iwahori-Hecke algebras of type B, J. reine angew. Mathematik 482 (1997), 191-213.

[Ge] B. Gemein, Singular braids and Markov's theorem, J. Knot Theory Ramifications 6, No. 4 (1997), $441-454$.

[GJKL1] D. Goundaroulis, J. Juyumaya, A. Kontogeorgis, S. Lambropoulou The Yokonuma-Temperley-Lieb Algebra, submitted for publication, arXiv:1012.1557.

[GJKL2] D. Goundaroulis, J. Juyumaya, A. Kontogeorgis, S. Lambropoulou Framization of the Temperley-Lieb algebra, in preparation. See also arXiv:1304.7440.

[Jo] V.F.R. Jones, Hecke algebra representations of braid groups and link polynomials, Ann. Math. 126 (1987), 335388.

[Ju1] J. Juyumaya, Sur les nouveaux générateurs de l algèbre de Hecke H(G,U,1), Journal of Algebra 204 (1998), 49-68.

[Ju2] J. Juyumaya, Markov trace on the Yokonuma-Hecke algebra, J. Knot Theory Ramifications 13, 25-39 (2004).

[JK] J. Juyumaya, S. S. Kannan, Braid relations in the Yokonuma-Hecke algebra, J. Algebra 239, No. 1 (2001), 272-297.

[JL1] J. Juyumaya and S. Lambropoulou, $p$-adic framed braids and p-adic Markov traces, Proceedings ITLD 2006, Hiroshima, Japan, Series on Knots and Everything, World Scientific Publ. Co. Ltd.(2007).

[JL2] J. Juyumaya, S. Lambropoulou, p-adic framed braids, Topology and its Applications 154 (2007), $1804-1826$.

[JL3] J. Juyumaya, S. Lambropoulou, An invariant for singular knots, J. Knot Theory Ramifications, 18, No. 6 (2009), 825-840.

[JL4] J. Juyumaya, S. Lambropoulou, An adelic extension of the Jones polynomial, M. Banagl, D. Vogel (eds.) The mathematics of knots, Contributions in the Mathematical and Computational Sciences, Vol. 1, Springer (2011).

[JL5] J. Juyumaya, S. Lambropoulou, p-adic framed braid II, Advances in Mathematics 234 (2013), $149-191$.

[JL6] J. Juyumaya, S. Lambropoulou, Modular Framization of the BMW algebra. See arXiv:1007.0092.

[Ka1] L.H. Kauffman, An invariant of regular isotopy, Transactions of the AMS 318, No. 2 (1990), $417-471$.

[Ka2] L.H. Kauffman, Virtual Knot Theory, European J. Combin. 20 (1999), 663-690.

[KL] L.H. Kauffman, S. Lambropoulou, Virtual braids and the L-move, J. Knot Theory Ramifications 15, No. 6 (2006), 773-811.

[KV] L.H. Kauffman, P. Vogel, Link polynomials and a graphical calculus, J. Knot Theory Ramifications 1 (1992), No. 1, 59-104.

[KS] K.H. Ko, L. Smolinsky, The framed braid group and 3-manifolds, Proceedings of the AMS, 115, No. 2 (1992), 541-551.

[La1] S. Lambropoulou, Solid torus links and Hecke algebras of B-type, in Quantum Topology; D.N. Yetter Ed.; World Scientific Press, 1994; pp. 225-245.

[La2] S. Lambropoulou, Knot theory related to generalized and cyclotomic Hecke algebras of type B, J. Knot Theory Ramifications 8, No. 5 (1999), 621-658. 
[Mu] J. Murakami, The Kauffman polynomial of links and representation theory, Osaka J. Math. 24 (1987), 745-758.

[OS] S.Yu. Orevkov, V.V. Shevchishin, Markov theorem for transversal links, J. Knot Theory Ramifications $\mathbf{1 2}$, No. 7 (2003), 905-913.

[PR] L. Paris, L. Rabenda, Singular Hecke algebras, Markov traces and HOMFLY-type invariants, Ann. Inst. Fourier (Grenoble) 58, No. 7 (2008), 2413-2443.

[Ra] S. Ramanujan, On Certain Trigonometric Sums and their Applications in the Theory of Numbers, Transactions of the Cambridge Philosophical Society 22, No 13 (1918), 297-276.

[Sm] L. Smolin, Knot theory, loop space and the diffeomorphism group. New perspectives in canonical gravity, 245-266, Monogr. Textbooks Phys. Sci. Lecture Notes, 5, Bibliopolis, Naples, 1988.

[TL] H.N.V. Temperley, E. H. Lieb, Relations between the 'percolation' and 'colouring' problem and other graphtheoretical problems associated with regular planar lattice: some exact results for the 'percolations problems', Proc. Roy. Soc. London Ser. A 322 (1971), 251-280.

[Th] N. Thiem, Unipotent Hecke algebras of $G L_{n}\left(\mathbb{F}_{q}\right)$, J. Algebra 284 (2005), 559-577.

[We] H. Wenzl, Braids and invariants of 3-manifolds, Invent. Math. 114 (1993), 235-275.

[Wr] N. Wrinkle, The Markov theorem for transverse knots, Preprint \protect \vrule width0pt \protect \href $\{\mathrm{http}: / / \mathrm{arxiv}$.org/abs

[Yo] T. Yokonuma, Sur la structure des anneaux de Hecke d'un groupe de Chevalley fini, C.R. Acad. Sc. Paris 264 (1967), 344-347.

J. Juyumaya: Instituto de Matemáticas, Universidad de Valparaíso, Gran Bretaña 1091, Valparaíso, CHILE.

E-mail address: juyumaya@uvach.cl

S. Lambropoulou: Department of Mathematics, National Technical University of Athens, ZoGrafou CAMpus, GR-157 80 Athens, Greece.

E-mail address: sofia@math.ntua.gr

$U R L:$ http://www.math.ntua.gr/ sofia 